\title{
Structural Characterisation of Image Processing Operators
}

\author{
P.Bottoni*, L.Cinque*, S.Levialdi*, P.Mussio ${ }^{+}$, B.Nebbia* \\ * - DSI, Università di Roma, Via Salaria 113,00198 Roma, Italy \\ +- DEA, Università di Brescia, Via Branze 38, 25231 Brescia
}

\begin{abstract}
We present a methodology to specify the action of image processing operators in terms of transformations induced on structures present in an image. The methodology is based on an analysis of the characteristics of the operator with respect to the adopted segmentation technique and is illustrated through an example of characterisation of the Gaussian filter.
\end{abstract}

\section{Introduction}

Automatic generation of image interpretation strategies is facilitated if descriptions of the behaviour of available operators exist specifying which structures of an image are affected and which remain unaltered by the operator application. It is thus possible to decide which operators to choose, based on descriptions of the image at hand, of the quality of results one wants to obtain and of the known features of available operators.

In particular, one is interested in using operators preserving relevant structural features. In this case, the usual characterisation of operator behaviours based on their action on numerical features of the image signal, such as noise or texture [1], is insufficient. For example, in restoring line drawings, one wants to sieve out triangular structures from the borders of a binary image, while preserving significant corners [2]. This method is here generalised to characterise image processing operators (IPOs) via the transformations they induce on structures defined in terms of their shape. Structures are first described as strings of attributed symbols identifying pixels in which the contour changes direction [3]. Shapes are then characterised by grouping these attributed symbols into more abstract shape descriptions, again expressed by attributed symbols [2]. An IPO effect is characterised by defining how descriptions of structures present in the input and output images are transformed.

The diagram of Fig.1 summarises this view. Given an image I, an IPO Tr maps it into a new image I'. The analysis algorithm anl [3] derives a description $D$ of image I and a description $D^{\prime}$ of I'. The problem is: find a description transformation? such that the diagram commutes (i.e. identical results are obtained by combining Tr and anl or anl and ?). We characterise $\mathrm{Tr}$ by specifying this transformation.

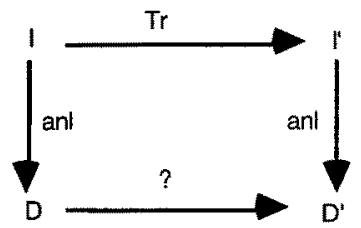

Fig. 1. Image and description transformations

Description transformations are here characterised by rewriting rules. We define an analytical methodology which extracts rules defining the transformations from numerical specification of the given operators. The main contribution of the paper is the proposal of a method for the characterisation of operators through explicit description of the shape transformations they perform, and the consequent definition of notations, based on run codes, to describe symmetrical structures, used for the experimental part. The methodology is designed to solve the problem of defining the behaviour of image operators for automatic generation of image processing and 
interpretation strategies; in particular, to assess which structures types are invariant for a transformation, and to characterise families of structures with respect to transformations under which they are invariant. The paper develops as follows. In Section 2 descriptions are introduced as strings of attributed symbols. Two attributes characterise structures based on a run-length encoding. Section 3 illustrates the method to characterise shape transformations, and Section 4 draws conclusions.

\section{Shape Characterisation}

A structural description of an image enumerates the structures recognised in it, with each structure described by a name - identifying its type and denoted by a symbol - and a vector of properties - the values assumed by a set of variables, called attributes. The n-tuple with the symbol and its properties is an attributed symbol. Structures can be decomposed into simpler ones. Primitive structures are not further decomposable.

Following [3], primitive structures for binary image description are black pixels, called multiple elements, in which the contour changes its direction locally. They are associated with the symbol ME with attributes: code, summarising the pixel's 8-neighbourhood, as in

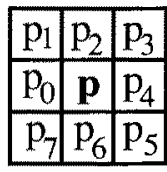

Fig. 2, and coordinates; code is: $\operatorname{code}(p)=\sum_{i=0}^{7} u_{i} \cdot 2^{i}$, where $u_{i}$ is 1 if the pixel $p_{i}$ is white, 0 if it is black.

Fig. 2. The ordering of 8 neighbourhoods

Hence, each multiple element is described by an attributed symbol $<M E$, code, coordinates $>$ and the whole image by the string of symbols describing its multiple elements. In [3] it is shown that such a description is sufficient to reconstruct any original black and white image. For example, Fig. 3 is an image in which white pixels are background, while textured and black pixels constitute the image. Textured pixels are the multiple elements. The whole image is described by the string of attributed symbols describing the seven MEs, Im: $\langle M E, 143,5,1\rangle\langle M E, 6,5,5\rangle$ $<\mathrm{ME}, 31,2,8><\mathrm{Me}, 12,8,14><\mathrm{Me}, 62,8,16><\mathrm{ME}, 248,9,16><\mathrm{ME}, 227,9,1>$. The MEs marked with $\mathrm{A}, \mathrm{B}$ and $\mathrm{C}$ describe a particular structure called, $r$-triangle which can be described by an attributed symbol <tri,base,length,fstpt>. A is associated with the code $31, \mathrm{~B}$ with the code 6 and $\mathrm{C}$ with code 12. A Conditional Attributed L-System with Interaction (CAIL) [2] can be defined (see Fig. 6), which: 1) identifies the substring $\mathrm{Tr}$ : $<M E, 6,5,5><M E, 31,2,8><M e, 12,8,14>$ in the string describing the image, 2) recognises that the three elements are vertices of a rectangle triangle, 3 ) evaluates the triangle attributes and 4) generates the new attributed symbol.

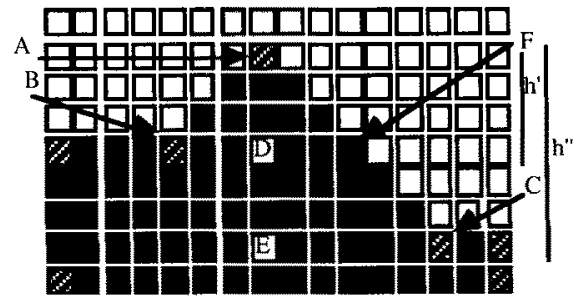

Fig.3. An upward triangle defined by the sequence of codes $(6,31,12)$. 
R-triangles are defined in geometrical terms and constitute a well defined set of contour structures in $b / w$ images. Analogously, the set of substrings describing these structures is a language on ME specified by a CAlL. In general, the set $V$ of types of structures is assumed as alphabet; each single structure is described by an attributed symbol, formed by a symbol in $V$, and by its property vector. An image description is a string of attributed symbols. Structure description based on MEs is formalised by CAILs. CAILs extend the grammars traditionally used in syntactic pattern recognition [4] in two main ways typical of L-systems [5]. First, they use one single alphabet, not partitioned in terminals and non-terminals. Each symbol denotes a structure, not a possible derivation of it. Second, direct generation is parallel.

A $C A I L$ is a rewriting system $R W=(V, P, \Rightarrow)$ and a semantic domain $D=(\Omega, \Phi, \Gamma)$. V is a finite set of symbols, the alphabet. $P$ is a finite set of productions of the form $<<\omega_{1}, \delta, \omega_{2}>, \varepsilon>$, meaning that for $\delta$ to be rewritten as $\varepsilon$, it must be embedded in the context represented by $\omega_{1}, \omega_{2} \in V^{*} \Omega=\left\{D_{1}, \ldots, D_{k}\right\}$ is a finite set of domains. $\Phi$ is a set of functions. $\Gamma$ is a set of predicates, also comprising the constant predicates true and false. For each $\mathrm{p} \in \mathrm{P} \exists \gamma \in \Gamma$, called the condition to be satisfied for $\mathrm{p}$ to apply, and $\exists r_{p} \subseteq \Phi$, a finite set of functions computing attributes of the consequent as a function of those of the antecedent. The rewriting relation $\Rightarrow$ specifies that rules in $P$ are applied in parallel to directly generate a string $Z$ from a string $W$ (in symbols $W \Rightarrow Z$ ).

Rules are applied to strings of attributed symbols. Strings are cyclical, so that two consecutive symbols describe two consecutive multiple elements in the contour. In the image, structures are set of pixels described by substrings of multiple elements.

A side is a set of contour pixels connecting two consecutive multiple elements. The side length is length $\left(\mathrm{p}_{1}, \mathrm{p}_{2}\right)=\max \left(\left|\mathrm{x}_{1}-\mathrm{x}_{2}\right|,\left|\mathrm{y}_{1}-\mathrm{y}_{2}\right|\right)$. Geometrical entities are also used as attributes to describe structures. For example, the symmetry axis of a structure is a line in the image splitting the structure into two isomorphic parts. A run is a sequence of adjacent black pixels orthogonal to a symmetry axis. In the following, we exploit possible partial symmetries of structures emerging from the contour of components to derive transformation laws of their descriptions. We consider contour structures for which an intuitive geometrical notion exists and provide their formal definition in terms of strings of attributed symbols, through suitable CAILs. CAILs are also used to specify how structures are modified by application of IPOs.

\subsection{Characterisation of Trapezes}

An $i$-trapeze, as shown in Fig. $4 \mathrm{a}$, is the digital version of an isosceles trapeze appearing on the contour of an image. The trapeze is delimited by the multiple elements marked with A,B,C,D. The upper base (upbase) is the sequence of pixels from the second to the third multiple element, while the lower base (lowbase) is the sequence of pixels from the first to the fourth, including these two. The height of an i-trapeze is the sequence of pixels from the second multiple element to the nearest pixel in the base. Similar trapezes can appear oriented in the eight directions and with different lengths of their upper base and of their height. However, for each direction and for each size, the four multiple elements delimiting the trapeze present a specific sequence of codes. For example, the one shown in Fig. $4 \mathrm{a}$ is characterised by the code sequence $(6,15,30,12)$, and size changes only affect the coordinates of the multiple elements in the description of the particular image. Hence, there is a finite number of possible sequences of codes for the multiple elements which can delimit an i-trapeze.

The set of descriptions of possible structures of type i-trapeze, denoted by the 
symbol itrpz, is defined by the rule in Fig. 5, where TRPZ $=\{(6,15,30,12)$, $(3,135,195,129),(192,255,240,96),(48,120,60,24),(12,30,15,6),(129,195,135,3)$, $(96,240,255,192),(24,60,120,48)\}$. This rule allows the recognition of description of i-trapezes and the substitution of the sequence of four elements denoting the delimited i-trapeze with a more synthetic attributed symbol itrpz, whose attributes lowbase, upbase and height and fstpt are computed by the semantic rules and allow the reconstruction of the trapeze.

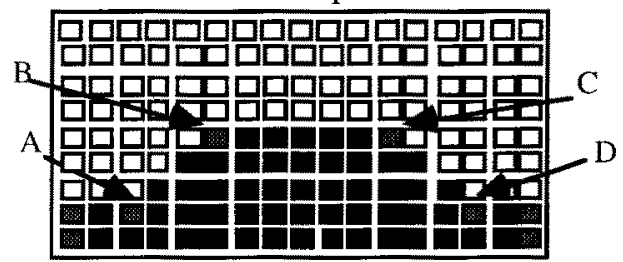

a)

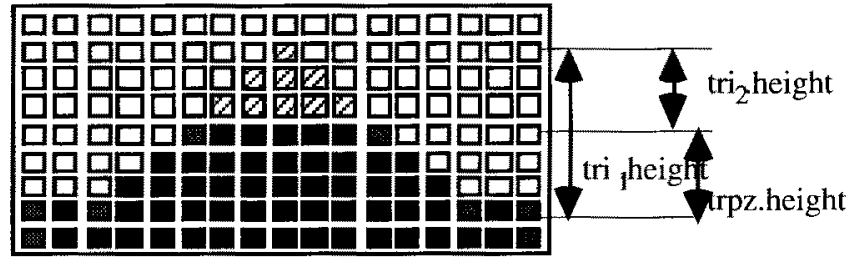

b)

Fig. 4. a) An i-trapeze on the contour; b) i-trapeze as difference of two isosceles triangles.

Tuples in TRPZ define the clockwise sequences of codes for extremal points of an $i$ trapeze. The construction in Fig. $4 \mathrm{~b}$ shows the relation among the attributes. In particular, note that a trapeze results from the difference of two triangles $\operatorname{tri}_{1}$ and $\operatorname{tri}_{2}$. Its height is thus defined by the law $\operatorname{trpz} . h=\operatorname{tri}_{1} \cdot h$-tri $i_{2} . h$. Since for a triangular structure $h=(b+1) / 2$, we obtain $\operatorname{tri}_{1}$. height $=(\operatorname{trpz}$.lowbase +1$) / 2$ and $\operatorname{tri}_{2}$. height $=[(\operatorname{trpz}$. base $2)+1] / 2$. Therefore: $\operatorname{trpz}$.height $=[(\operatorname{trpz}$.lowbase +1$) / 2]-\{[(\operatorname{trpz}$.upbase -2$)+1] / 2\}=$ $($ trpz.lowbase-trpz.upbase+2)/2.

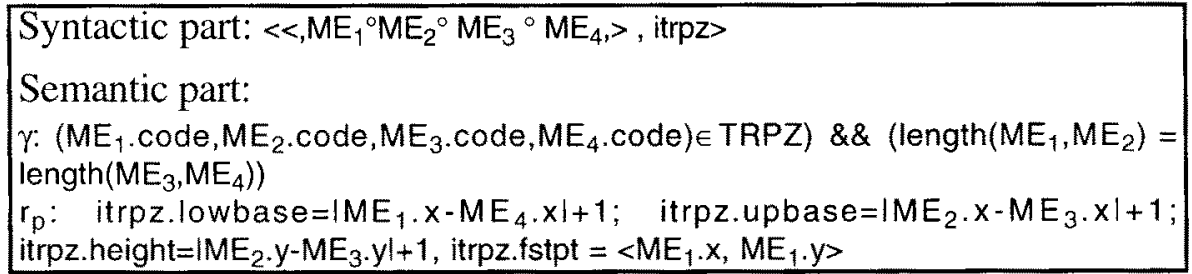

Fig. 5. The rule for defining a trapeze.

\subsection{Characterisation of Rectangle Triangles}

In a similar way, an r-triangle, as the one shown in Fig. 3 , is the digital version of a rectangular triangle. The base of an r-triangle is the sequence of pixels from the first to the third multiple element, including these two. The height of an r-triangle is the sequence of pixels from the second multiple element to the nearest pixel in the base. Again, the fstpt attribute defines the initial coordinates of the triangle. 


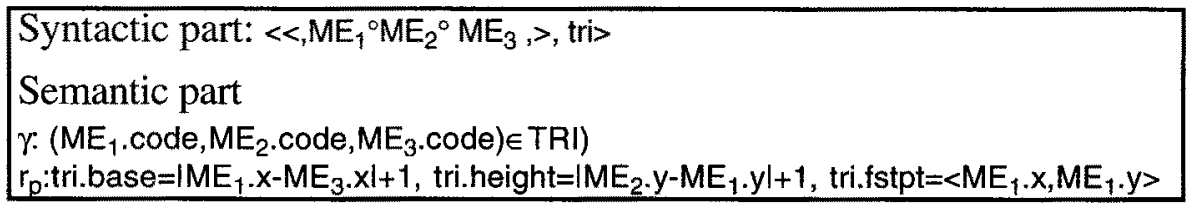

Fig. 6. The rule for defining an r-triangle

The set of descriptions of possible structures of type r-triangle, denoted by the symbol tri, is defined by the rule in Fig. 6 , where TRI $=\{(6,31,12),(3,199,129)$, $(192,241,96),(48,124,24),(12,31,6),(129,199,3),(96,241,192),(24,124,48)\}$. Triples in TRI define the clockwise sequences of codes for extremal points of an $r$-triangle. Such a triangle can be seen as the superposition of a symmetrical triangle on a rectangle trapeze. For example, in Fig. 3, the pixel marked with $\mathrm{A}$ is the vertex of the symmetrical triangle which extends between vertices $B$ and $F$, while the trapeze has bases DF and EC respectively. $h^{\prime}$ and $h^{\prime \prime}$ are the heights of these triangles. We call symmetrical component the substructure delimited by the pixels $\mathrm{B}, \mathrm{A}$ and $\mathrm{F}$, and residual component the substructure delimited by pixels $\mathrm{F}$ and $\mathrm{C}$.

The relation holds tri.base $=h^{\prime}+h^{\prime \prime}-1$. For Fig. 3, tri.base $=10, h^{\prime}=4, h^{\prime \prime}=7$. The semantic rule $r_{p}$ is used in the case of upwards and downwards triangles. The case for leftwards and rightwards triangles is derived by exchanging the $\mathrm{x}$ and $\mathrm{y}$ coordinates. Constraints exist on base and height of an r-triangle and on their relations. In particular, the minimum height is 2 and the minimum base is 3 . Due to the structure partial symmetry, for each pixel added to the height $h$ ', the base is increased of two pixels, for each pixel added to the height $h^{\prime \prime}-h^{\prime}$, the base is increased of one pixel.

\subsection{Description of runs}

Structures of interest are characterised through a description of the runs composing them. The study of the variations of the run-lengths of structures helps to derive rules defining the transformation of a structure into another, by considering the independent transformations of symmetrical and non-symmetrical components of structures, based on a run-length encoding. Indeed, each structure in which the sides maintain their orientation can be seen as the composition of a symmetrical structure with an asymmetrical one. We call such structures oriented structures. Each component is formed by a sequence of parallel runs. With each run a number is associated representing its length. Hence an oriented structure is characterised by the sequence of lengths of its runs. We call such a sequence a length-sequence, and it becomes the value of an attribute lenseq for symbols denoting oriented structures.

Theorem 1: For each type $T$ of oriented structure there is a language $L(T)$ of strings of natural numbers, coding the sequences of lengths, each string uniquely describing a structure of type $T$ up to localisation.

The proof is based on the following lemma.

Lemma 1 A symmetrical structure is composed only of runs of the same parity.

Based on these definitions, a function is derived for computing the attribute lenseq associated with an oriented structure. For example, for $r$-triangles the function trilen depends on the two heights $h^{\prime}$ and $h^{\prime \prime}$ of the composing triangles and is inductively defined as follows: trilen $\left(1, h^{\prime \prime}\right)=\Delta 102^{\circ} \ldots{ }^{\circ} h^{\prime \prime}$, trilen $\left(h^{\prime}, h^{\prime \prime}\right)=\Delta$ sym(trilen $\left.\left(h^{\prime}-1, h^{\prime \prime}\right)\right)^{\circ}\left(2 h^{\prime}-\right.$ 1) $02 h^{\circ} \circ . .{ }^{\circ}\left(h^{\prime}+h^{\prime \prime}-1\right)$, where the function sym extracts the subsequence between the two separators $\Delta$ and $\vartheta$. The symbol ${ }^{101}$ denotes concatenation, ' $\theta$ ' is a separator between the 
symmetrical and the residual components. Note that, following the contour clockwise, the description of residual component comes after the separator 0 . Had this component been before the symmetrical part, its description would have preceded the separator $\Delta$. In the following, we develop the argument only for structures where the residual component follows the symmetrical component. The inverse case is easily derived.

As an example, the r-triangle of Fig. 3 is described by the string of naturals, $\operatorname{tri}(4,7)=\Delta 1^{\circ} 3^{\circ} 5^{\circ} 7 \circ 8^{\circ} 9^{\circ} 10$, where each number represents the length of a run and 1 denotes the vertex of the r-triangle, considered as a run of length one; 3 and 5 are the lengths of the following runs, 7 is the length of the base of the symmetrical component, and the sequence $8^{\circ} 9^{\circ} 10$ describes the length of the runs of the residual components.

Another useful notation associates an oriented structure with the variation-sequence in the length of its runs. This sequence is the value for an attribute varseq.

Theorem 2: For each type $T$ of oriented structure there is a language $L(T)$ of strings of natural numbers, coding the variations in length of the runs, each string uniquely describing a structure of type $T$ up to the localisation and length of the top run.

As an example: $\operatorname{tri}(4,7)=\Delta 1^{\circ} 3^{\circ} 5^{\circ} 7 \diamond 8^{\circ} 9^{\circ} 10 \equiv \Delta 2 ; 2 ; 2 \diamond 1 ; 1 ; 1=\Delta 2^{3} \diamond 1^{3}$, where ';' is now used to denote concatenation. In general, for an r-triangle of heights $h^{\prime}$ and $h^{\prime \prime}$ the

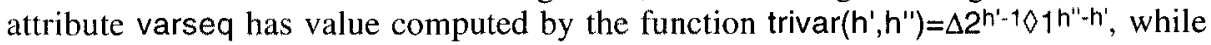
for an i-trapeze of height $h$, the attribute is calculated as $\operatorname{trpz} \operatorname{var}(h)=\Delta 2^{h-1} \nabla$. Note that symbols in the strings associated with symmetrical components are even numbers.

\section{Characterising Shape Transformations}

In this Section we illustrate the methodology for analytical extraction of rewriting rules, using as example the transformations induced by a Gaussian filter [6], in the case that the segmentation operator is a thresholding.

We use a Gaussian filter with kernel of size 5, and we consider the same size for the neighbourhood of pixels. The convolution kernel is symmetrical and generated from the vector $1 / 20 \times[1,5,8,5,1]$, through the law: $w(i, j)=w(i) \times w(j)$. Due to the symmetry characteristics of both the filter and the considered structures, it is possible to identify some minimum values for the size of the structures, in order for the transformation to be characterised by a regular, formalisable behaviour. In a binary image, there are four cases for the centre pixel of a neighbourhood: 1) internal pixel, 2) pixel close to background, 3) pixel close to foreground, and 4) background pixel.

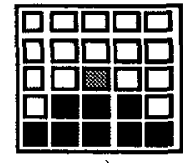

a)

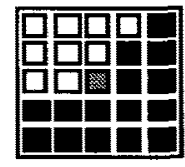

d)

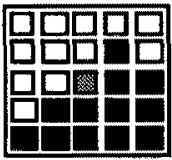

b)

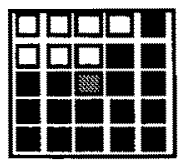

e)

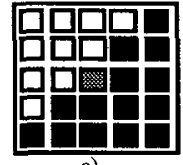

c)

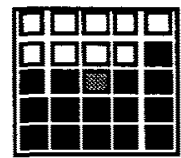

f)

Fig. 7. Some configurations of neighbourhoods on the contour of an r-triangle. 
We refer to grey level images with values in a set $\{0, \ldots, 255\}$. The segmentation algorithm is a simple thresholding. Here we adopt as threshold the value 200 ; a pixel of coordinates $(n, m)$ is transformed into a pixel with grey value 255 if and only if formula 1 is satisfied, where $w(i, j)$ denotes the weight of the element of position $(i, j)$ in the convolution kernel and $[g(n+i, m+j)=255]$ denotes the elements with value 255 .

$$
W=\left(\sum_{i, j \in\{-2,+2\}} w(i, j) \cdot[g(n+i, m+j)=255]\right) \geq \frac{200}{255}=0.7843
$$

To define the effect of the filtering on the segmented image, neighbourhood configurations are analysed to identify those allowing the central pixel to be transformed into 255. Fig. 7 shows neighbourhoods on the contour of an r-triangle.

\subsection{Transformation of an $r$-triangle}

The method described above is here used for the case of the r-triangle. In Fig. 8a, A indicates from which pixel all pixels maintain their value while descending toward the base. In Fig. 8b, B indicates until which pixel the value is maintained.

For the neighbourhoods considered in Fig. 7, the central pixel is transformed into 0 . All the other pixels in the r-triangle which are close to the background are instead transformed into 255. This result is extended to neighbourhoods resulting from reflection and rotation of the neighbourhoods above. There exists a minimal height, viz. 4 pixels, above which a general law can be identified. Below this height, the methodology can describe the behaviour of the transformation for the single cases.

Theorem 3 (r-triangle). The variation-sequence associated with an $r$-triangle of heights $h^{\prime} \geq 4, h^{\prime \prime} \geq h^{\prime}+2$ is transformed by a Gaussian filter in a variation-sequence, by the law: $\Delta 2^{h^{\prime}-1} \diamond 1^{h^{\prime \prime}-h^{\prime}} \rightarrow \Delta 2^{h^{\prime}-2} \diamond 1^{h^{\prime \prime}-h^{\prime}}$.

《<segm, r-tri, segm $2>$, trpz $>$

$\gamma$ : (tri.height $\geq 4) \& \&$ (segm 1 . length $\geq 3$ ) \&\& (segm 2. length $\geq 3)$ )

rp: trpz.height'=tri.height'-1; trpz.height"=tri.height"-1; trpz.varseq=g(tri.varseq);

The overall structure transformation erases the r-triangle vertex, while leaving the same base length. Hence, an r-triangle symmetrical part is transformed into a trapeze, while the residual component maintains its size, but with a lower base run. The structural transformation induced by the Gaussian filter is summarised by the following rule, with $g$ defined by Theorem 3 , and trpz a symbol denoting a trapezoidal structure with the same sequence of codes as an i-trapeze, but different side lengths. As an example, for the case of Fig. 9 we have: $G[(\operatorname{tri}(5,7))]=\Delta 2^{4} \Delta 1^{2}$.
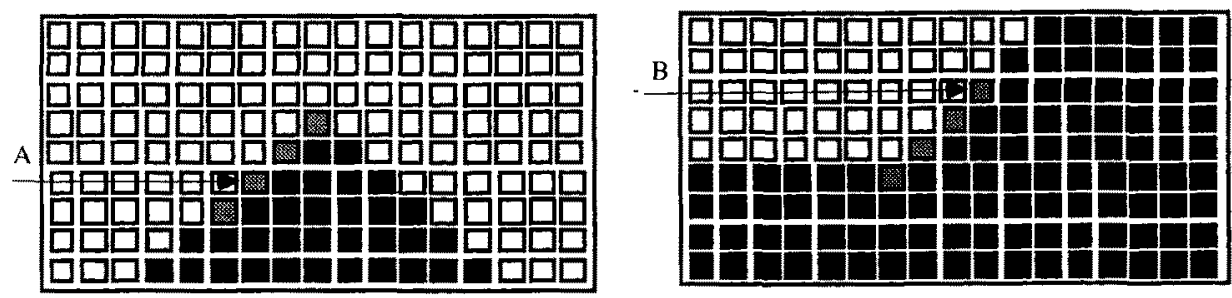

Fig. 8. Initial (a) and final (b) configurations on the contour of an r-triangle

The transformation law for variation sequences in i-trapezes is analogous to that for 
triangles, but the structure size does not change, since the overall effect is that of lowering it. Hence i-trapezes are invariant under iteration of Gaussian filtering (until there are sufficient pixels in the whole object).

Theorem 4 (i-trapeze). The variation-sequence associated with an i-trapeze of height $h \geq 4$ and upbase $>1$ is transformed by a Gaussian filter as: $2^{h-1} \rightarrow 2^{h-1}$

The CAIL for trapeze transformation derives, with the semantic rule including $\operatorname{trpz} z_{1}$. lowbase $=\operatorname{trp} z_{2}$.lowbase; $\operatorname{trp} z_{1}$. upbase $=\operatorname{trp} z_{2}$. upbase; $\operatorname{trp} z_{1}$. height $=\operatorname{trp} z_{2}$. height.
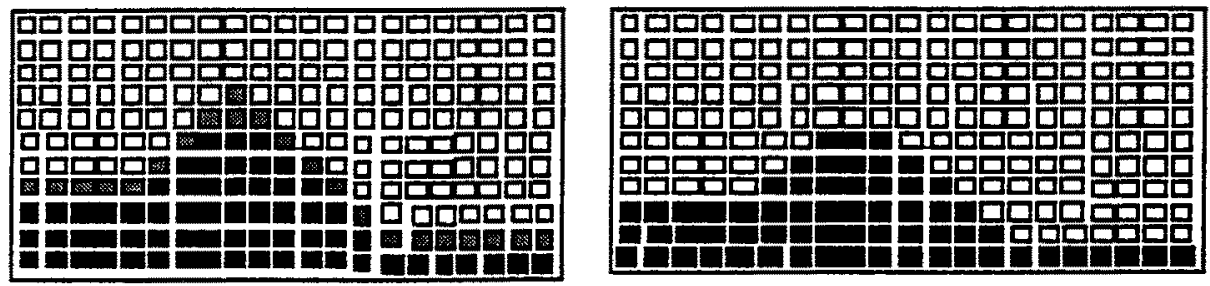

Fig. 9. Gaussian transformation of an r-triangle.

\section{Conclusions}

We have extended the structural approach to image description via rewriting rules to the problem of describing transformations of structures in an image, under application of global image transformations. The methodology is based on an analysis of the characteristics of the operator with respect to the adopted segmentation technique.

The conditions for its application are that structures are composed of a symmetrical part and of a residual, non-symmetrical, but regular-i.e. maintaining its orientation, component. Both components must consist of contiguous pixels along a contour. Moreover, the structure size must be sufficient to avoid high-frequency effects.

This research aims at providing symbolic descriptions of the characteristics of IPOs, in order to facilitate their automatic selection during the generation of an image interpretation strategy. This will require defining a language for operator description and a metalevel interpreter able to select suitable operators based on such descriptions.

\section{References}

[1] C.Spinu, C.Garbay, J.M.Chassery, "A cooperative and adaptive approach to medical image segmentation", in P.Barahona, M.Stefanelli, J.Wyatt eds., Artificial Intelligence in Medicine, Springer, 1995, 379-390.

[2] P.Bottoni, P.Mussio, M.Protti, R.Schettini, "Knowledge-based contextual recognition and sieving of digital images", PRL., 10(2):101-110, 1989

[3] P.Mussio, D.Merelli, M.Padula, "An approach to Definition, Description and Extraction of structures in Binary Digital Images", CVG\&IP, 31:19-49. 1985.

[4] K.S.Fu, Syntactic Pattern Recognition and Applications, Prentice-Hall, 1982.

[5] G.T.Herman, H.Rozenberg, Developmental Systems and Languages, NorthHolland, 1975.

[6] P.J.Burt, "Smart sensing within a pyramid vision machine", Proc. of the IEEE, 76(8):1006-1015, 1988. 